\title{
Z-Selective hydrofunctionalization of dienes
}

Clara Vicera ${ }^{1}$, Raphael Dada ${ }^{1}$, Rylan J. Lundgren ${ }^{1}$

${ }^{1}$ Department of Chemistry, University of Alberta

\begin{abstract}
Olefins play a fundamental role in synthetic organic chemistry because they are useful building blocks that create molecules. However, geometry control (E- vs Z-) in olefin synthesis is of utmost importance because the olefin geometry has a tremendous impact on its physical, chemical and biological properties. Additionally, Z-olefins are less stable compared to their Eolefin counterparts; due to this difference, general methods to make olefins results in more cases of E-olefins production with relatively fewer Z-olefins caused by its instability. It has been reported that Z-olefins can be synthesized from dienes through a rhodium-catalyzed formate mediated transformation, with tolerance to several reducible functional groups. With this successful method in hand, the focus is to make functionalized Z-alkenes while still maintaining tolerance to reducible functional groups under mild reaction conditions.

Thus, this project presents the production of Z-olefins through rhodium-catalyzed hydrofunctionalization using the starting materials, dienes and aldehydes. This method requires an inert atmosphere and the reaction progress can be monitored by Nuclear Magnetic Resonance (NMR) using an internal standard to quantify the amount of product formed. In this process, it was observed that the starting material was consumed until more than $95 \%$ conversion. In addition, the possibility of using different dienes, such as diene esters and phenyl dienes, as well as different aldehydes could further broaden the scope of this method. The usefulness of this process can be applied to the production of complex molecules. For example, in the synthesis of a glucagon receptor antagonist, which is a drug that is used in the treatment of diabetes. Currently, there is a limited number of methods used to create Z-olefins; however, this proven procedure can be further applied in other hydrofunctionalization experiments.
\end{abstract}

Key words:

Hydrofunctionalization of dienes, Z-Selective, Reductive Coupling, Rh-Catalysis, Z-Homoallylic Alcohols

Cite as: Vicera C., Dada R., Lundgren RJ. 2019. Z-Selective Hydrofunctionalization of Dienes. Alberta Academic Review, Vol 2 (2) 77-78, WISEST Special Issue (non peer-reviewed), DOI 10.29173/aar74. 
Vicera et al., 2019

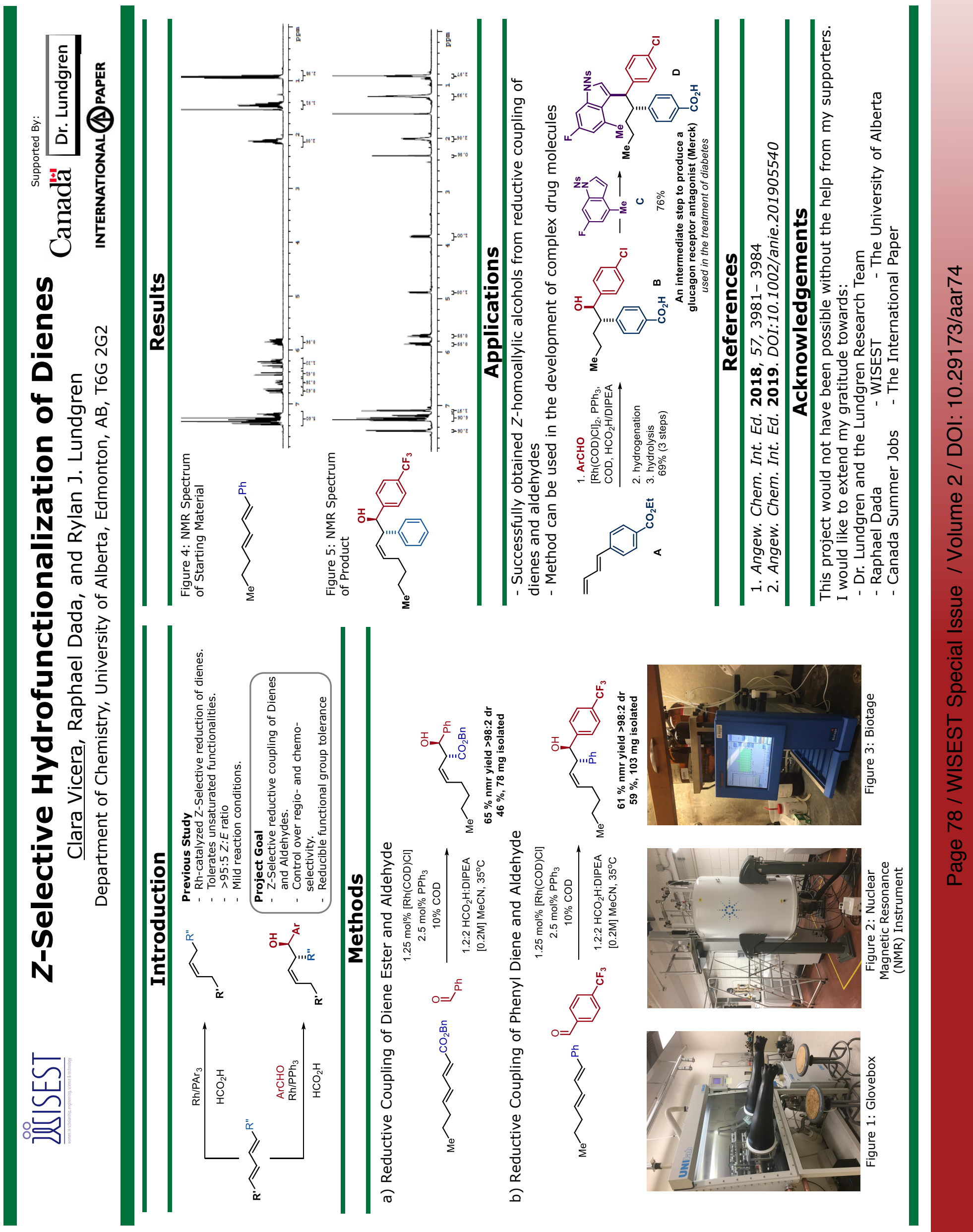

